\title{
A performance analysis on packet scheduling schemes based on an exponential rule for real-time traffic in LTE
}

\author{
Ee Mae Ang ${ }^{1}$, KuokKwee Wee ${ }^{1 *}$, Ying Han Pang ${ }^{1}$ and Keat Keong Phang ${ }^{2}$
}

\begin{abstract}
Long-Term Evolution (LTE) was implemented to fulfill and satisfy users' needs as well as their demands for an improvised, fast and efficient Quality of service (QoS). A minimal aggregate of waiting time in return would give users a better Quality of experience (QoE). Real-time service packet scheduling is an important process in allocating resources to users. An efficient packet scheduling scheme will be able to cater fairly and efficiently to its users in the LTE network. Hence, studies are performed focusing on real-time traffic which includes video as well as Voice over Internet Protocol (VolP) transmissions. In this work, the existing exponential rule (EXP rule) is utilized to benchmark our proposed packet scheduling techniques so that we are able to further evaluate the scheduling performance. In response to the increasing likelihood of losing packets in the EXP rule's algorithm and maximizing the throughput rate, several schemes have been experimented with. The proposed schemes include 1) simplified EXP rule (sEXP Rule), 2) modified EXP rule (mEXP Rule), 3) EXP rule with maximum throughput (MT) (EXP_MT Rule), and 4) enhanced EXP rule with MT (E2M). By adding MT as a weight to the EXP rule, the throughput is maximized, thus providing higher throughput rates for real-time and non-real-time traffic. The simulation results show that the sEXP rule has a better performance in throughput, packet loss rate (PLR), and spectral efficiency for video traffic. Aside from this, our proposed E2M rule performs better than the benchmark EXP rule and outperforms the other proposed schemes, as well. It is observed that the E2M rule has better QoS support for real-time transmission in terms of delay, packet loss, throughput and spectral efficiency, within the LTE network. Hence, our proposed E2M rule is an enhancement of the benchmark EXP rule, which is commonly used in LTE packet scheduling.
\end{abstract}

Keywords: Downlink; Scheduler; LTE network; Quality of service; Exponential function; Real-time transmission

\section{Introduction}

The future fourth generation wireless network, which is known as the Long-Term Evolution (LTE) network, supports devices which utilize high-speed data, and at the same time it targets to provide users with better wireless network experience while maintaining minimal complexity [1]. Transmissions are categorized into real-time and non-real-time traffic. Real-time traffic includes video and Voice over Internet Protocol (VoIP) traffic, whereas non-real-time traffic consists of best-effort traffic.

People are looking forward to more advanced devices that can meet their desires and needs. A survey in [2]

\footnotetext{
* Correspondence: wee.kuok.kwee@mmu.edu.my

'Faculty of Information Science and Technology, Multimedia University, Jalan Ayer Keroh Lama, Bukit Beruang, Malacca 75450, Malaysia

Full list of author information is available at the end of the article
}

mentioned that in several upcoming years, mobile data's growth is anticipated to double yearly. Based on the increasing demand of the Internet Protocol-based services on the wireless network like Voice over Internet Protocol (VoIP), website browsing, video streaming, online games and etc., requirements of the Quality of service (QoS) has to be assured as it is of utmost importance in the wireless network [3-5]. The functionality of QoS is rather appealing in metrics computation, which is used for packet scheduling in the media access control (MAC) layer as well as packet routing in the physical layer $[6,7]$. This is especially so for real-time traffic QoS demands which include the requirement of having a lower packet loss rate (PLR) and delay, whereas nonreal-time traffic QoS demands are more geared toward throughput rate. However, despite the fact that resource 
allocation is handled in MAC layer, there is no guarantee that data has been successfully transmitted and received [8]. Hence, the evolution of managing radio resource distribution is necessary in various scenarios in the LTE environment [9]. At present, a network engineer's tasks tend to be more challenging in terms of managing networks resources [7].

In contrast to satisfying users' demands and serving all users fairly, the LTE network was developed to provide much more reliable performance in terms of its spectral efficiency, fairness measures, throughput rate, less idle time, and minimal PLR [10]. Packet schedulers play a role in the MAC layer of the LTE environment. Hence, its function is to select and schedule packets based on the QoS requirements $[11,12]$. For example, QoS is used to determine performance trade-offs [13, 14]. Traffic scheduling is associated with channel management to attain high-performance networks, which guarantee QoS requirements of prioritized real-time traffic [15].

This paper emphasizes the packet scheduling schemes for real-time applications in LTE systems' network, inclusive of channel-aware/QoS-aware strategies and channelaware/QoS-unaware strategies. Our focus is on prioritizing real-time applications using the channel-aware/QoS-aware strategies. Based on our studies, we have chosen the exponential (EXP) rule, which is most commonly referenced by researchers due to the fact that this scheme is able to guarantee delay requirements and it is aware of channels conditions as well as QoS. In order to determine the reliability of the packet scheduling scheme, the QoS performance is evaluated. Although the EXP rule is a popular scheme, we have observed that there is still room to improve it in terms of its throughput, PLR, and delay. As mentioned above, our focus is on real-time services as these are more challenging compared to serving non-real-time services. Real-time services include video and VoIP traffics. Apart from this, this study also addresses the issue of heavy traffic, including real-time services, which require less delay and PLR in order to receive the information in good quality on time. Priority should be given to real-time services, which is then followed by the non-real-time services.

Our paper proposes several packet scheduling techniques for the LTE network based on the EXP function. Currently, it can be seen that the EXP function is able to exaggerate a value and hence prioritize it [16]. The EXP rule is not only robust in terms of its growth compared to the EXP/proportional fairness (PF) rule packet scheduling scheme, but is also an enhancement based on EXP/PF rule [10]. The EXP rule is known for having low delay and a high measure of fairness as well as throughput [17]. Furthermore, while the EXP rule takes account of the comprehensive network status, it does not consider packets that are lost due to the packet expiration dateline.
Simulations are performed prior to downlink scheduling in the LTE network. Downlink scheduling is whereby packets are scheduled to transmit from eNodeB (eNB) to user equipments (UE) $[9,18]$. Based on the simulation results, it has been demonstrated that our proposed algorithm for packet scheduling is able to provide a better QoS performance, especially for multimedia applications. This in return will raise user satisfaction as users' Quality of experience (QoE) increases [19]. Our algorithm not only provides a more reliable wireless network, but also a more efficient one, which is thus able to satisfy users' demands. Our proposed scheme is able to increase the chance of packets being sent within the time frame, ensuring less delay and PLR, while having a higher throughput rate. This is especially for real-time applications, such as live video. One of the several schemes we have proposed outperforms the existing benchmark EXP rule, i.e., the enhanced EXP rule with MT (E2M) rule, as it contributes to a much lower PLR, and delay while providing an increment in the system's throughput value. Essentially, real-time packets are prioritized before the packet expiration dateline. More details are provided in Section II.

This paper is arranged as follows: Section II gives a brief elaboration on the existing schemes available for LTE networks packet scheduling. In Section III, a detailed elaboration on our proposed schemes is given. Section IV provides an overview of the simulation parameters that are used to carry out the experiments. In addition, the results that are obtained from the experimental test are provided in the same section, along with a brief discussion based on our findings. In the last section, we conclude the paper.

\section{Literature review}

This section briefly elaborates on several downlink scheduling schemes, which have been proposed by other researchers and also frequently used for the analysis and comparison of LTE networks. These schemes have similar strategies, whereby they are aware of their channels conditions. Table 1 shows several notations along with their representations.

\section{A. Proportional fairness (PF)}

A PF scheduler is able to provide fairness in allocating resource blocks (RB) to users. Additionally, it is capable of providing an efficient amount of bandwidth in its network [10]. This scheduler makes full use of the networks throughput in order to provide a good level of fairness among its users. PF is able to achieve this by monitoring the channel's quality as well as the previous user's throughput value $[11,20]$. 
Table 1 Notations for scheduling metrics

\begin{tabular}{|c|c|}
\hline Expression & Implication \\
\hline$\overline{m_{i, k}}$ & Common metric for the $i^{\text {th }}$ user's $k^{\text {th }}$ resource block \\
\hline$d_{k}^{i}(t)$ & The attained data rate by the $i^{\text {th }}$ user at the $t^{\text {th }}$ period \\
\hline $\bar{R}^{i}(t)$ & $\begin{array}{l}\text { The } i^{\text {th }} \text { user's attained regular amount of throughput until } \\
\text { time } t\end{array}$ \\
\hline$D_{H O L, i}$ & The head-of-line delay of the $i^{\text {th }}$ packet \\
\hline$\tau_{i}$ & The threshold delay of the $i^{\text {th }}$ user \\
\hline$w_{i, k}$ & $\begin{array}{l}\text { The channel's weighted spectral efficiency for the } i^{\text {th }} \text { user in } \\
\text { the } k^{\text {th }} \text { resource block }\end{array}$ \\
\hline$\delta_{i}$ & The reasonable amount of packet loss by the $i^{\text {th }}$ user \\
\hline$N_{R T}$ & Number of real-time traffic flow \\
\hline
\end{tabular}

In [10] and [21], it is shown that PF is suitable to schedule best-effort (BE) traffic. By paying attention to the channel's quality with regard to the preceding user's throughput rate, PF is able to prioritize channels that have a much better traffic constraint from the source $[22,23]$.The metric for PF is given as $m_{i, k}^{P F}$. It is expressed as shown in Eq. 1 [10]:

$$
m_{i, k}^{P F}=\frac{d_{k}^{i}(t)}{\bar{R}^{i}(t-1)}
$$

where $d_{k}^{i}(t)$ is the attainable amount of data, and $\bar{R}^{i}$ $(t-1)$ is the forecasted attainable throughput rate at the period of the $i^{\text {th }}$ transmission time interval (TTI) that is anticipated for the $i^{\text {th }}$ user equipment grounded by the total amount of bandwidth along with its respective $R B$ [10]. The disadvantage of this scheme is that it incapable of prioritizing traffic with critical conditions [24]. Hence, traffic may encounter an amount of delay which may lead to packet loss if packets are not served before packet expiry [25]. Moreover, PF is also unable to provide the highest throughput nor best fairness measure.

\section{B. Maximum throughput (MT)}

This scheduling technique falls under the same classification as PF, which is a channel-aware but QoSunaware strategy [11]. In [26], it is stated that MT is able to exploit overall available resources to attain a higher throughput rate in the network's system. Throughout the fixed TTI, the server shall transmit a maximum number of packets to the terminal [27]. The MT's metric, $m_{i, k}^{M T}$, is shown in Eq. $2[21,26]$ :

$$
m_{i, k}^{M T}=d_{k}^{i}(t)
$$

where the MT's metric is equivalent to the attainable amount of information at the $i^{t h}$ user for the $k^{\text {th }} \mathrm{RB}$. The signal-to-interference-plus-noise ratio (SINR) is used in the computation of the throughput value. The expression is shown in Eq. 3 [10]:

$$
d_{k}^{i}(t)=\log \left[1+\operatorname{SINR}_{k}^{i}(t)\right]
$$

MT is unable to provide fairness measures in terms of resource sharing [28]. Packets that consist of a lowpriority level may undergo resource starvation when the requested requirements, based on the overall bandwidth, are unsatisfied $[21,28]$.

\section{Modified largest weighted delay first (M-LWDF)}

M-LWDF is an extension of LWDF [10]. LWDF is a channel-unaware scheduling scheme which has been modified to become aware of its channels conditions and provisions for delays in bounded packet transmissions. M-LWDF is channel-aware/QoS-aware and is able to provide good performance in terms of fairness measures, spectral efficiency, and less PLR. Moreover, in [29], the authors mention that this scheme has the best performance in video transmissions during UE movement at a minimal speed.

In the implementation of this scheme, the method distinguishes between non-real-time and real-time traffic. This is in order for the real-time packets to be able to be prioritized, using the packets head-of-line (HOL) delay. The metric is computed as described in Eq. 4 [11]:

$$
m_{i, k}^{M-L W D F}=\beta_{i} D_{H O L, i} m_{i, k}^{P F}
$$

where $\beta_{i}$ is computed by the mathematical equation $\frac{\log \delta_{i}}{\tau_{i}}$, in which it is functioned to weigh two flows which consists of the same HOL delay. Its decision is based on legitimate requirements of acceptable PLR and packet expiry deadlines [10].

In the MAC queue, packets which are an associate of real-time services and have not been transmitted by the time of its expiry date shall be removed from the queue [11]. This is performed to avoid the consumption of bandwidth.

\section{Exponential/proportional fairness rule (EXP/PF rule)}

According to [4], the EXP/PF rule was implemented with the purpose of offering QoS promises over a shared wireless connection. The basic EXP was created to improve the real-time flow's priority prior to the association of non-real-time flows [30,31]. This is where the packet delay at the starting line is rather close to the thresholds delay [10]. EXP/PF not only guarantees a bounded delay but also maximizes the systems throughput 
at the same time ensuring proportional fairness between UEs [32].

The scheduling computation metric's computation is given in Eq. 5 [11]:

$$
m_{i, k}^{E X P / P F}=\exp \left(\frac{\alpha_{i} D_{H O L, i}-x}{1+\sqrt{x}}\right) m_{i, k}^{P F}
$$

The symbol $x$ computation is given in Eq. 6, where $\alpha_{i}^{\prime}$ s value is given as an element of the set $\left[\frac{5}{\left(0.99 \tau_{i}\right)}, \frac{10}{0.99 \tau_{i}}\right] \cdot \tau_{i}$ symbolizes the packet's waiting time's threshold value. $D_{H O L, i}$ represents the delay for HOL at the $i^{\text {th }}$ user, and $m_{i, k}^{P F}$, explained in Eq. 2, acts as the weight for the metric:

$$
x=\frac{1}{N_{r t}} \sum_{i=1}^{N_{r t}} \alpha_{i} D_{H O L, i}
$$

The symbol $N_{r t}$ is the value of real-time flows which are active in the downlink. In [31], analysis showed that $\mathrm{EXP} / \mathrm{PF}$ is able to support the performance on real-time traffics in terms of average throughput and PLR, although a portion of the system throughput is forfeited.

\section{E. Logarithm rule (LOG rule)}

The LOG rule contributes in giving much more efficient QoS support to the network by maintaining an average amount of delay. In [4], it was stated that to some degree this scheme's approach in allocating resources, is identical to the EXP rule, which is elaborated on in the next section. It functions to exploit the structures throughput whereby the traffic arrival as well as the condition of the channels is known. The computation metric for the LOG rule is given as $m_{i, k}^{L O G}$ Rule in Eq. 7 [10, 33]:

$$
m_{i, k}^{L O G ~ R u l e}=b_{i} \log \left(c+\alpha_{i} D_{H O L, i}\right) \Gamma_{k}^{i}
$$

Several parameters in Eq. 7 are adjustable, including $\alpha_{i}, b_{i}$, and $c$. As for $\Gamma_{k}^{i}$, it signifies the channels spectral efficiency with the $i^{t h}$ user at the $k^{t h} \mathrm{RB}$, where $1 \leq i \leq N$.

However, based on a test performed by [10], it was found that by declaring these constraints, given in Eq. 8, the scheduling performance is much better.

$$
\alpha_{\mathrm{i}}=\left[\frac{5}{\left(0.99 \tau_{i}\right)}\right], b_{i}=\frac{1}{E\left[\Gamma^{i}\right]}, c=1.1
$$

Arithmetically, the EXP and logarithm functionality is somewhat similar. The variance is that in which the logarithmic function implements the inverse performance of the EXP function [34].

\section{Proposed methodology}

The packet scheduling schemes are studied using the LTE-Simulator, which is also known as LTE-Sim [35]. It is an open-source simulator which enables the performance of simulations in an LTE network [11].

Both the EXP rule and the M-LWDF scheduler are bounded delay schemes. The EXP rule is an improvisation of the aforementioned EXP/PF scheduler [10]. The EXP rule is known to be more robust based on its growth and the fact that it monitors the entire network's status. Hence, it is categorized under the channel-aware and QoS-aware strategy for packet scheduling. [10] stated that every user's delay is normalized against the overall amount of delay that has been experienced by all the users.

The metric for the EXP Rule, $m_{i, k}^{E X P}$ Rule, is given in Eq. 9 [10, 36]:

$$
m_{i, k}^{E X P R \text { Rule }}=\mathrm{b}_{\mathrm{i}} \exp \left(\frac{\alpha_{i} D_{H O L, i}}{c+\sqrt{\left(\frac{1}{N_{R T}}\right) \sum_{j} D_{H O L, j}}}\right) \Gamma_{k}^{i}
$$

where a set of fixed constraint's which include $\alpha_{i}, b_{i}$, and $c$ are provided in (10). The constraint's values are the same as the Log Rule's parameters in Section II:

$$
\alpha_{i} \in\left[\frac{5}{\left(0.99 \tau_{i}\right)}, \frac{10}{\left(0.99 \tau_{i}\right)}\right], b_{i}=\frac{1}{E\left[\Gamma^{i}\right]}, c=1
$$

Based on some indirect measures, the EXP rule scheduler is found to have improvements in delay, fairness measures, and throughput [37, 38]. In [11], it was stated that this scheme prioritizes real-time traffic with regard to non-real-time traffic. In short, it supports both application services [39]. Similarly to M-LWDF, packets that are not served before their expiration date shall be removed from the queue.

Based on experimental tests, the EXP rule outperforms the most frequently used M-LWDF scheduling scheme in terms of delay and PLR in real-time traffic. Hence, further research has been performed on the EXP Rule scheduling scheme.

In the following subsections, several packet scheduling schemes are proposed. This includes the EXP with MT rule (EXP_MT rule), the simplified EXP rule (sEXP rule), the modified EXP rule (mEXP rule), and the enhanced EXP rule with MT (E2M rule).

\section{A. Simplified EXP rule (sEXP rule)}

As its name implies, the sEXP rule is a simplified version of the EXP rule whereby the denominator is removed to increase the metrics value. The sEXP rule is designed to reduce the complexity of computation. 
Hence, the sEXP rule's metric, $m_{i, k}^{s E X P}$ Rule, is left with the mathematical equation given in Eq. 11:

$$
m_{i, k}^{s E X P \text { rule }}=\exp \left(D_{H O L, i}\right) m_{i, k}^{P F}
$$

As mentioned above, the denominator of the EXP rule (refer to Eq. 6), which consists of a computation on the average HOL delay, is taken out due to the fact that it does not show much significance as it is canceled out by the HOL delay at the numerator of the EXP rule. Adjustable numerical figures, such as the fixed constraints given by the author in [10], are not included in our proposed SEXP rule.

\section{B. Modified exponential rule (mEXP rule)}

The mEXP rule is based on the existing EXP rule and somewhat similar to the proposed sEXP rule. mEXP consists of several optimal parameters which are absent in the EXP rule's equation, as well as its numerator, which makes it distinguishable from the EXP rule. As for the difference between the mEXP rule and the sEXP rule, this is where there is an additional element inserted into the mathematical expression. This would increase the metrics value as well as the metrics priority. After modifying the expression, the mEXP rule's metric, $m_{i, k}^{m E X P}$ Rule, is given as shown in Eq. 12:

$$
m_{i, k}^{m E X P \text { rule }}=\exp \left(\gamma_{i} D_{H O L, i}\right) m_{i, k}^{P F}
$$

where the equation of $\gamma_{i}$ is almost similar to $\beta_{i}$, which is applied in the M-LWDF scheduling scheme $[10,20]$. Hence, the modified version is given as shown in Eq. 13:

$$
\gamma_{i}=-\ln \left(\frac{\delta_{i}}{0.99\left(\tau_{i}\right)}\right)
$$

The $\delta_{i}$ 's value is an integer with 6 as its value, based on the optimal parameter that was suggested in [10]'s work. The probability value for packet loss has been increased by replacing log with the ln function. This is so that the chance of losing a packet is smaller.

\section{EXP rule with MT (EXP_MT rule)}

Based on the EXP rule computation in Eq. 6, a change to the metrics weight is made. Instead of using PF, MT is used as a substitute in this scheme in order to provide the channel with a higher throughput during packet transmission, thus reducing the fairness measures [10, 20]. As mentioned above, MT functions to maximize the throughput value.

The mathematical computation of the EXP_MT rule's metric, $m_{i, k}^{E X P_{M} T}$ Rule , is expressed in Eq. 14:

$$
m_{i, k}^{E X P_{M} T \text { Rule }}=\exp \frac{\alpha_{i} D_{H O L, i}}{c+\sqrt{\left(\frac{1}{N_{R T}}\right) \sum_{j} D_{H O L, j}}} m_{i, k}^{M T}
$$

where, with reference to parameter $\alpha_{i}$ of Eq. 6, where $\delta_{i}$ is an integer with a value of 6 , whereas $c$ is an integer with value 1 , as it is mentioned that the values are fixed as such to provide a much more efficient scheduling performance. $m_{i, k}^{M T}$ is the MT scheme is used as the weighting factor. As mentioned above, our objective is toward real-time services. MT has the ability to obtain a higher significance in the amount of traffic delivered [10] compared with PF which provides fairness measures and efficient use of bandwidth. We assume that if more packets were delivered, this would mean less packet loss, thus contributing to better QoE and QoS.

\section{Enhanced EXP rule with MT (E2M rule)}

The E2M rule scheduler is derived from the EXP rule, the EXP_MT rule, and the mEXP rule. This scheme utilizes the EXP function, at the same time maximizing the packet transmission's throughput rate at the same time using an $\ln$ function which increases the probability of packet being loss. This is performed by applying the EXP rule with MT as the metric's weight, and also $\gamma_{i}$ as mentioned previously in Eq. 13. Hence, Eq. 15 shows the mathematical computation of the E2M's metric, $m_{i, k}^{E 2 M}$.

$$
m_{i, k}^{E 2 M}=\exp \frac{\gamma_{i} D_{H O L, i}}{c+\sqrt{\left(\frac{1}{N_{R T}}\right) \sum_{j} D_{H O L, j}}} m_{i, k}^{M T}
$$

By using $\gamma_{i}$ to increase the probability of the packet being lost, fewer packets will be lost, which in turn increases the priority of the packets to be delivered before the packets' expiration date. This will improve the packet scheduling's QoS performance. The improvement is in regard to several QoS parameters in relation to real-time services, which include PLR, delay, and throughput rate.

\section{Simulation results}

In the LTE-Sim [11], stated that the value of the metric determines which packet is to be scheduled first and so on. Metrics consisting of a greater value are to have a higher prioritization and be scheduled first, and are then followed by the next highest metric value and so on. This is performed until each and every single packet on the list that is to be scheduled is served [11].

\section{A. Simulation parameters}

Several parameters are set to perform simulation experiments and analysis of the scheduling schemes. 
Table 2 Simulation parameters

\begin{tabular}{ll}
\hline Parameters & Values \\
\hline PHY & OFDMA \\
$\begin{array}{l}\text { Bandwidth/frame } \\
\text { length }\end{array}$ & $5 \mathrm{MHz} / 10 \mathrm{~ms}$ \\
Frame structure & TDD with uplink-downlink configuration number 1, \\
& and periodicity of 5 ms. \\
Modulation & QAM, 4-QAM, 16-QAM \\
$\begin{array}{l}\text { Simulation } \\
\text { scenario }\end{array}$ & Single cell with an interference \\
$\begin{array}{l}\text { Simulation } \\
\text { duration }\end{array}$ & $60 \mathrm{~s}$ \\
Traffic models & Real-time: video and VOIP; non-real-time: best-effort \\
Mobility & eNodeB: constant position; UE: random direction \\
Speed & 3 km/h \\
Number of UEs & $5-70$ \\
UE's Interval & 5 \\
Video data & 128 kbps/ foreman_H264 data \\
rate/traffic & Generated by G.729 codec, an audio data \\
VolP traffic & compression scheme \\
\hline
\end{tabular}

Each transmission consists of video, VoIP,and BE traffic $[35,40]$. An average result from the five sets of simulations is obtained. Further details of the parameters customized for the simulation are stated in Table 2.

\section{B. Results and discussion}

Based on our findings, the EXP rule outperforms the most frequently used M-LWDF scheduling scheme in terms of delay and PLR for real-time services. Thus, the results that were obtained from the simulations of our proposed schemes were analyzed in comparison to the existing EXP rule scheduling algorithm which we use as our benchmark scheme. Hence, our findings are deduced as follows.

As seen in Fig. 1, the EXP_MT rule, E2M, and sEXP rule have less PLR from 20 UEs onwards for video traffic. In addition, the EXP_MT rule outperforms all the scheduling schemes in terms of PLR for video traffic starting from 20 UEs onwards which has a packet loss ranging from 0.26 to $16.23 \%$. The percentage of change is computed and ranges from 60.49 to $99.08 \%$. E2M is the second best algorithm, as, when compared with the existing EXP rule, there are improvements in term of PLR from 20 UEs onwards that vary in a percentage ranging from approximately 30 to $90 \%$. In the sEXP rule, its performance outperforms the EXP rule from the count of 5 UEs right up to 70 UEs. However, the percentage of change is not as drastic as that of the EXP_MT rule, as it ranges from 0 to approximately $39 \%$. Furthermore, it is observed that for all schemes, PLR increases as the number of UEs increases.

Figure 2 illustrates the PLR for VoIP traffic in the LTE network. According to the figure, it can be observed that PLR gradually rises as the number of UEs increases. Overall, it can be seen that there is a slight improvement in terms of the traffic loss of packets for the sEXP rule scheme in comparison to the EXP rule. This is especially so for 50 to 70 UEs where the improvements change in percentage ranges in between 4.69 and $38.96 \%$. The highest change in percentage would be at 35 UEs with a change of $50 \%$. In addition to that, at 35 UEs, the PLR is the lowest in the case of the sEXP rule with an approximate PLR value of $0.01 \%$. In fact, based on the results that were obtained, the SEXP rule and the EXP rule PLR values lie below $0.22 \%$ and $0.32 \%$, respectively.

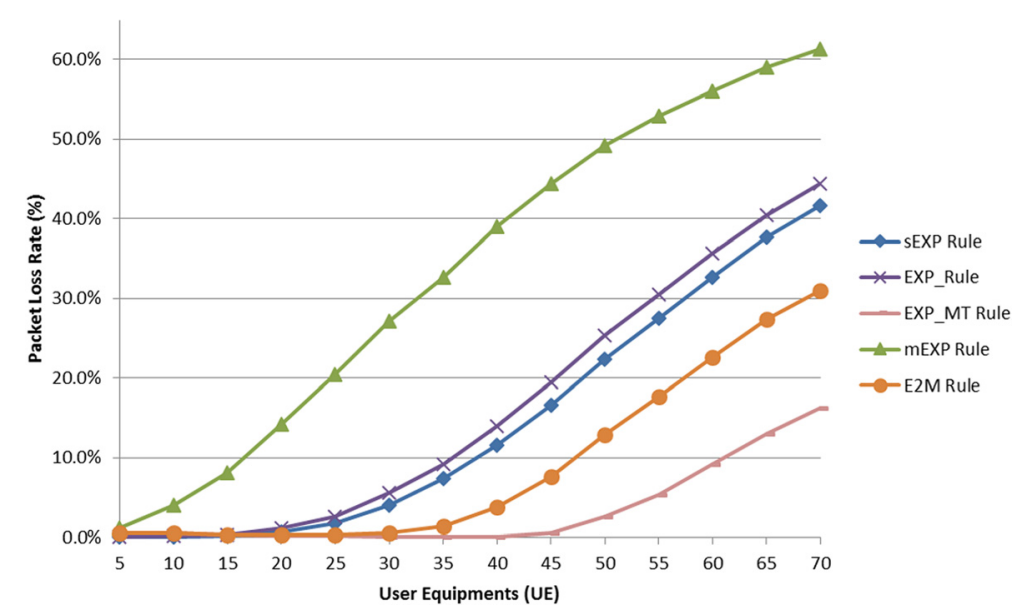

Fig. 1 PLR for video transmission 


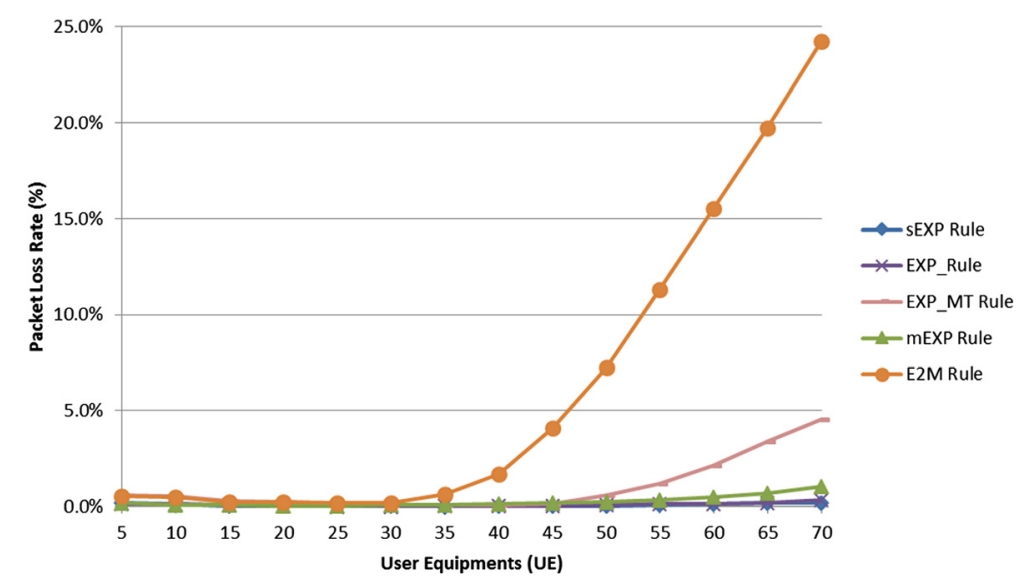

Fig. 2 PLR for VolP transmission

However, in regards to the mEXP rule, the EXP_MT rule, and the E2M, the percentage of change is rather drastic as many packets are lost during VoIP packet transmission. One of the reasons that packets are lost is due to overflowing queues. In addition, the output quality in VoIP transmissions is determined by the number of packets sent on time. Hence, packets that arrive late are meaningless to the user.

Figure 3 shows the PLR for BE traffic. Based on the results that were obtained, E2M outperforms the EXP rule by having less packet loss from 45 to 70 UEs with a change in percentage ranging between 0.6 and $3.6 \%$. The reason for this is that in E2M an increase in PLR probability ensures that packets are sent before their expiry. As for the sEXP rule, the percentage of change fluctuates as the number of users increases. The highest PLR is $3.2 \%$ for 10 UEs, while the lowest PLR is $2.8 \%$ for 65 UEs. In the case of the EXP_MT rule, the PLR is slightly higher than the benchmark EXP rule with a change in percentage that ranges between 0.20 and $6.66 \%$ as packets are discarded due to their lapse period. However, the mEXP rule has a much lower percentage of change in PLR compared to the EXP_MT rule, as the difference in change is from 0 to $3.83 \%$.

In the communication channel, the proportion of successful packets sent for the video transmission has to satisfy the users need to obtain the information. As can be seen, throughput for the mEXP rule scheduler is the lowest of all the schemes. This is due to the fact that many packets are loss during video transmission, which in turn, assigns the resources to the BE. On the other hand, the sEXP rule, E2M, and the EXP_MT rule schemes have much better throughput performance in contrast to the EXP rule. Comparing the sEXP rule, as shown in Fig. 4, the throughput result is slightly higher than that for the EXP rule. The gap between the sEXP rule and the EXP rule has a percentage of change of which the highest is $8.12 \%$ at 50 UEs. Both the sEXP

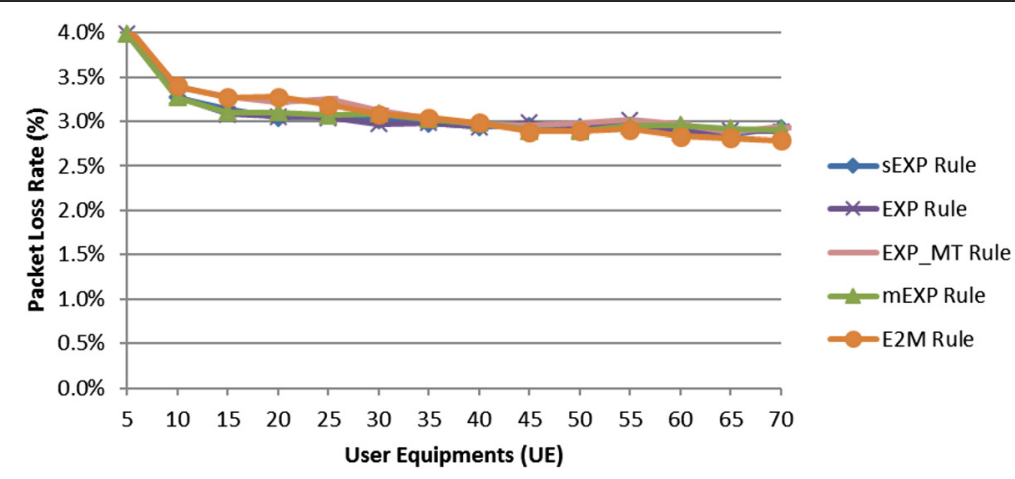

Fig. 3 PLR for BE transmission 


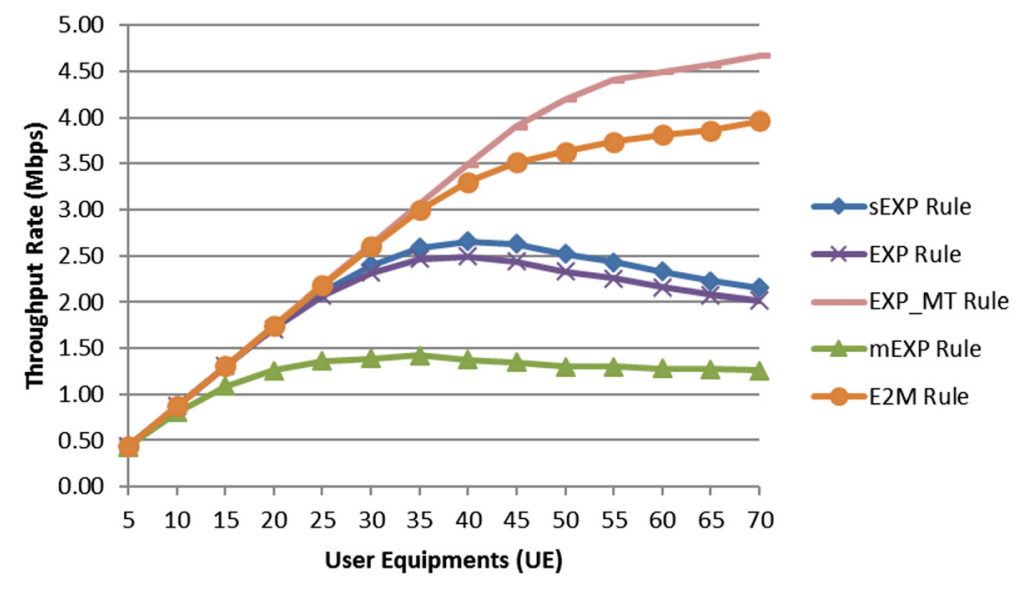

Fig. 4 Throughput for video transmission

rules and the EXP rules throughput rate drops little by little starting from 40 UEs onwards. The next scheme above the sEXP rule is the E2M rule, which has an increasing progress in its throughput rate from 15 UEs onwards. The incremental value's percentage of change, when compared to the existing scheme, ranges from 0.04 to $96.85 \%$. Lastly, the EXP_MT rule consists of a much better throughput rate compared to all the schemes, and therefore the best throughput rate for video transmission. Both E2M and the EXP_MT rule uses MT as their weight, thus enabling full utilization of the networks throughput. Similarly to the E2M rule, the rate increases as the number of UEs increases. In contrast to the existing EXP rule scheme, the EXP_MT rules percentage of change lies between 0.07 and $131.98 \%$. The throughput rate is affected by the PLR rate, hence the higher the amount of packet loss, the fewer packets there are transmitted over the communication channel (and vice versa).
In Fig. 5, VoIP throughput rises steadily with some very minor fluctuations as the number of UEs increases. Based on the variation in percentage calculations, changes are less than $7 \%$ for the sEXP rule, the EXP_MT rule, and the mEXP rule relative to the EXP rule. The sEXP rule has an improvement in throughput rate between 0.7 and $2.73 \%$ in terms of percentage changes from 25 to 50 UEs. As for the E2M rule, Fig. 5 illustrates that from 40 to 70 UEs, the throughput rate increases little by little, unlike the other experimented scheduling schemes. In addition to the E2M rule, the E2M rule has a slightly better throughput rate from 10 to 35 UEs, showing a change between 0.16 and $2.83 \%$ compared to EXP rule.

The amount of successful packet transmission for $\mathrm{BE}$ is computed and illustrated in Fig. 6. Based on the illustration, it can be observed that all of the proposed schemes outperform the EXP rule scheduling scheme. Just above the EXP rule is the sEXP rule, where the

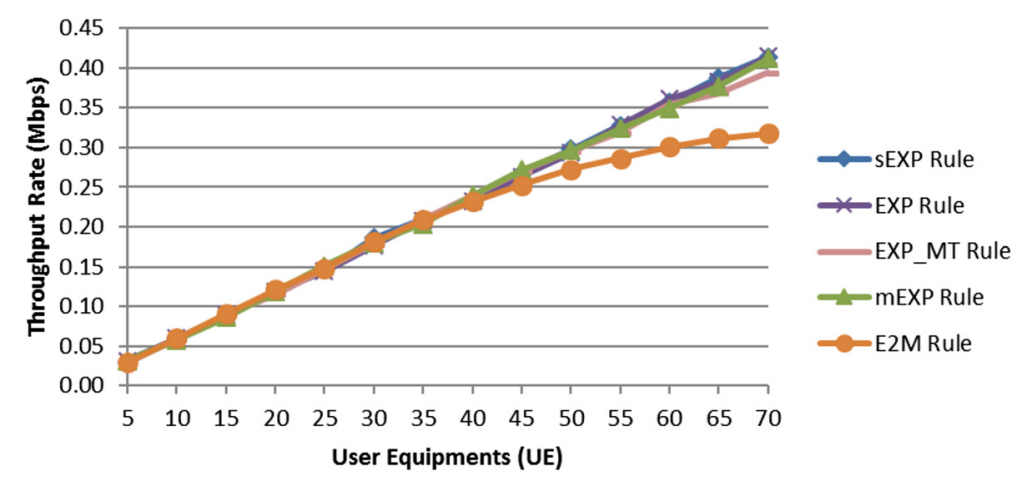

Fig. 5 Throughput for VolP transmission 


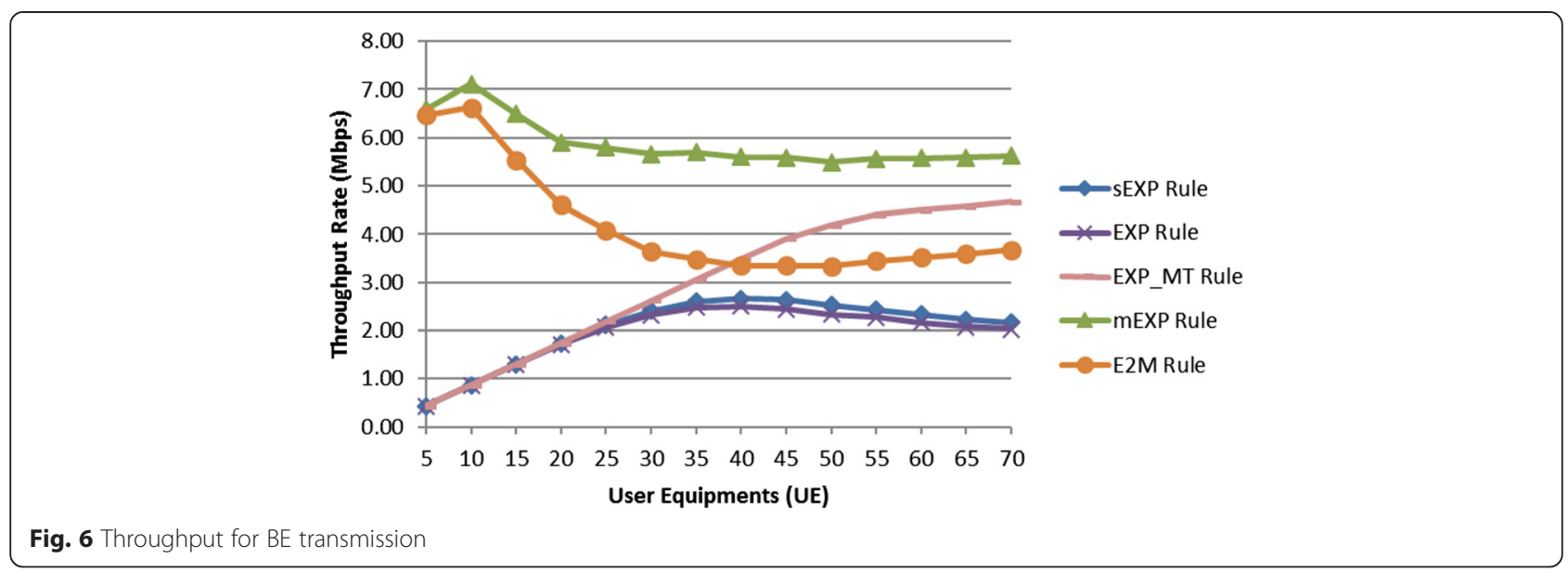

throughput value ranges between approximately 0.4 and $2.2 \mathrm{Mbps}$, and the percentage of change increases from 5 to 50 UEs. Its changes vary from 0.02 to $8.04 \%$. As for the EXP_MT rule, the throughput gradually increases as the number of UEs increases. The throughput rate ranges between 0.4 and slightly below $5.0 \mathrm{Mbps}$ for 5 to 70 UEs. In the E2M rule and the mEXP rule, the throughput rate slightly decreases as the number of UEs increases throughout the simulation process. At the point of approximately 40 UEs, the EXP_MT rule supersedes the E2M rule with a difference of less than 1 Mbps. Overall, the mEXP rule shows the best throughput performance for the $\mathrm{BE}$ traffic transmission despite the gradual decrease in throughput value. Using PF for its weighting helps to provide fairness in distributing resources among its users. It throughput rate is somewhat consistent, between approximately 3 and 7 Mbps. This is due to the small amount of packet loss and constant delay threshold value in the $\mathrm{BE}$ traffic.
In Fig. 7, video transmission delay is illustrated. The lowest amount of delay is with the E2M rule. It outperforms not only the existing EXP rule, but also the other proposed schemes, as well. In addition, it is fairly consistent as the delay is between 0 and $0.01 \mathrm{~s}$. In contrast with the EXP rule, the E2M rule has an enhancement of approximately 35 to $84 \%$. This may be due to the fact that there is a sudden increase in PLR at 30 UEs onwards in the video transmission due to congestion, which in turn contributes to a slight increase in delay. The EXP_MT rule has a lower delay than our benchmark EXP rule scheduler, especially from 5 to 50 UEs. From 5 to 15 UEs, the delay for the EXP_MT rule is below the E2M rule, with a change of 0.25 to $1.33 \%$. However, there is sudden spike in delay from 30 UEs onwards due to the throughput rate being prioritized which leads to the loss of packets as the number of UEs grows bigger. This is due to there being more packets in the queue that are waiting to be sent successfully. Initially,

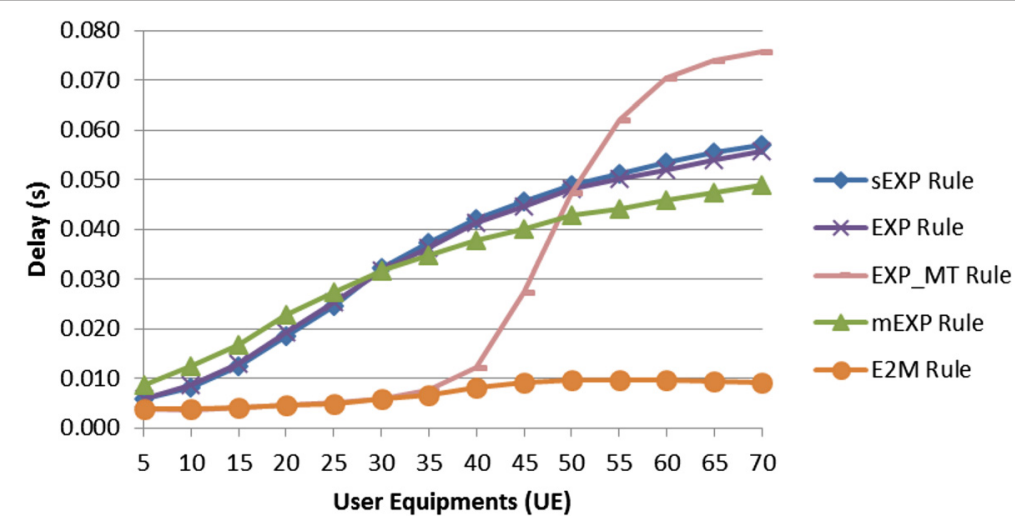

Fig. 7 Delay for Video transmission 


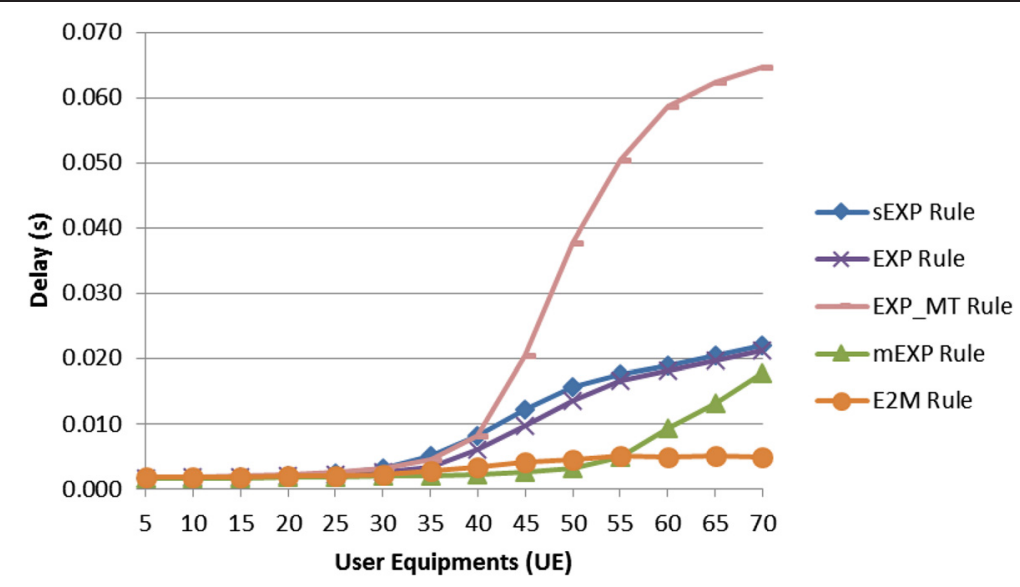

Fig. 8 Delay for VolP transmission

the mEXP rule's delay is higher than the benchmark scheduler, which is until the 30 UEs. In between 30 to 70 UEs, the delay gradually falls to a minimum, indicating that improvement lies between of 4 and $12 \%$. The sEXP rule is initially much better than the benchmark at a very small change in percentage which ranges between 2 and $5 \%$ from 5 to 25 UEs. However, from 30 UEs onwards there is no significant improvement as the delay increases between differences of 1 to $3 \%$. Hence, it can clearly be seen that the E2M rule has an outstanding result in terms of delay in video transmission. Packets are sent before their expiry dateline, in return reducing delay time as well.

Figure 8 illustrates the amount of delay obtained from the VoIP transmission. The delay in the EXP_MT rule is the highest compared to other schemes, followed by the sEXP rule. Both schemes have a higher amount of delay in contrast to the benchmark EXP rule. Even though the PLR soars for the EXP_MT rule from 40 UE onwards, as can be seen the delay has a steep curve. This is due to the fact that priority is given to the video transmission. Based on the figure, it is derived that the mEXP rule and the E2M rule have much better performance in terms of the amount of delay these schemes face. This is due to them having a combination of higher probability of packet being loss and HOL delay as a guide. Compared to the benchmark EXP rule, the mEXP rule's change in percentage initially increases from 5 to 50 UEs in an approximate range of 2.41 to $76.66 \%$. The amount of delay then begins to rise, which then gradually decreases the change in percentage as the number of UEs increases. The most striking change can be seen at 50 UEs where the proportion variation is the highest with a value of $76.66 \%$. As for the E2M rule, the delay remains

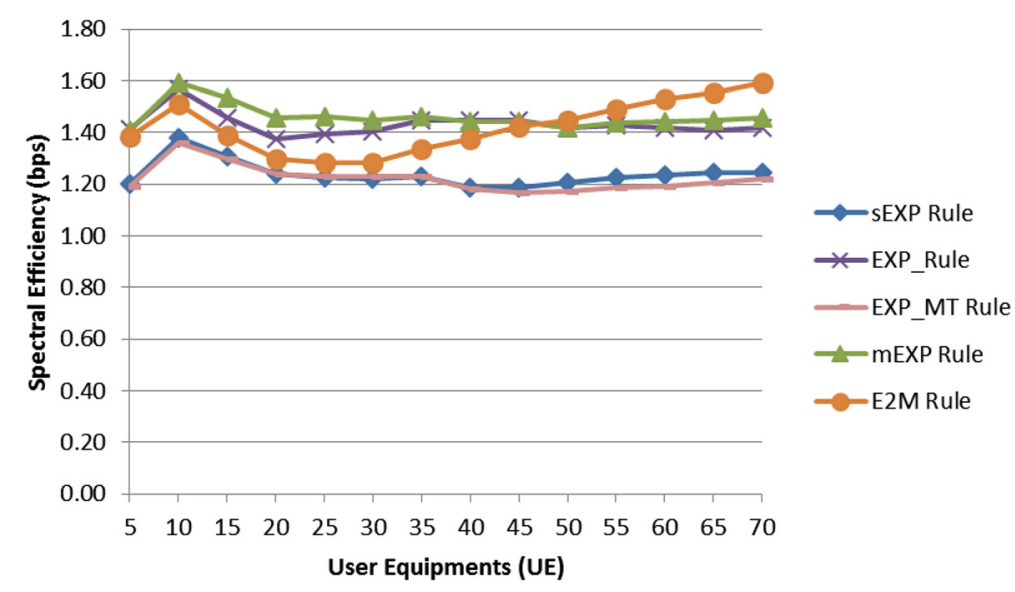

Fig. 9 Spectral efficiency 
Table 3 Experimental results overview

\begin{tabular}{|c|c|c|c|c|c|c|}
\hline \multirow[b]{2}{*}{ Schemes } & & \multicolumn{5}{|c|}{ QoS Factors } \\
\hline & & Delay & PLR & Throughput & Fairness & $\begin{array}{l}\text { Spectral } \\
\text { efficiency }\end{array}$ \\
\hline \multirow[t]{3}{*}{ EXP_MT rule } & VolP & $D$ & I & IS & IS & $D$ \\
\hline & Video & IS & I & I & I & \\
\hline & $\mathrm{BE}$ & - & D & I & IS & \\
\hline \multirow[t]{3}{*}{ sEXP rule } & VolP & D & I & IS & IS & D \\
\hline & Video & D & I & I & I & \\
\hline & $\mathrm{BE}$ & - & is & I & is & \\
\hline \multirow[t]{3}{*}{ mEXP rule } & VolP & I & D & IS & IS & I \\
\hline & Video & IS & D & $\mathrm{D}$ & $D$ & \\
\hline & $\mathrm{BE}$ & - & D & I & IS & \\
\hline \multirow[t]{3}{*}{ E2M rule } & VolP & I & D & IS & $D$ & IS \\
\hline & Video & I & I & I & IS & \\
\hline & $\mathrm{BE}$ & - & IS & I & D & \\
\hline
\end{tabular}

Symbol representation

I improved, IS improve slightly, $D$ degraded

averagely consistent between 0.001 to $0.005 \mathrm{~s}$ from 5 to 70 UEs. This is due to the increasingly large number of packets that are lost from 35 UEs onwards.

Figure 9 illustrates the spectral efficiency of the communication channel during the transmission flows. It can be seen that the mEXP rule transmits information at a faster rate over the given bandwidth of $5 \mathrm{MHz}$ in comparison to the benchmark EXP rule at UEs ranging from 10 to 35 and 55 to 70 . The highest bandwidth efficiency is at approximately $1.59 \mathrm{bps}$. This is succeeded by mEXP for 10 UEs, and the E2M rule for 70 UEs, respectively. The E2M rule starts with a lower spectral efficiency, below the benchmark rule, but then rises from 50 UEs onwards with a change ranging from approximately 1 to $13 \%$. However, the sEXP and EXP_MT rule have a lower bandwidth efficiency as can be seen in the Fig. 9 where these schemes are situated below the benchmark rule.

\section{Conclusions}

The focus of this study is on real-time applications, which includes video and VoIP traffic transmission. Based on the findings, the E2M rule has a much better performance compared to the other four proposed scheduling schemes, the existing EXP rule (which was used as the benchmark), the sEXP rule, the mEXP rule, and the EXP_MT rule which were tested in a simulation. The E2M rule stands out in terms of PLR, throughput rate, and delay. This is the case for realtime application, especially for video traffic flows. The E2M rule also has the highest efficiency in bandwidth from the 50 UEs onwards. This is followed by the EXP_MT rule, which has very good throughput values in $\mathrm{BE}$, video and VoIP traffic flows. The simulation results show that the sEXP rule has better performance in throughput, PLR, and spectral efficiency for video traffic. Lastly, the mEXP rule shows improvements in terms of delay for all traffic flows. An overview of the obtained results is given in Table 3 . The symbol "I" represents improvement, "IS" for slight improvement, and "D" for degraded performance, respectively. Thus, it can be concluded that the E2M rule outperforms the benchmark EXP rule as well as the other proposed schemes. Apart from that, based on the results and observations, the E2M rule is suitable for real-time traffic, especially for the video traffic transmissions.

\section{Competing interests}

The authors declare that they have no competing interests.

\section{Authors' contributions}

EMA proposed the scheduling algorithms, conducted simulation experiments, simulation analysis and drafted the paper. KKW participated in research supervision and direction,contributed in discussion and interpretation of the simulation results, as well as revising the manuscript. YHP has participated in equation analysis, mathematical computation in the simulation. KKP contributed in simulation model design and development, besides the traffic modelling. All authors have read and approved the final manuscript.

\section{Author details}

${ }^{1}$ Faculty of Information Science and Technology, Multimedia University, Jalan Ayer Keroh Lama, Bukit Beruang, Malacca 75450, Malaysia. ${ }^{2}$ Department of Computer System \& Technology, Faculty of Computer Science \& Information Technology Building, University of Malaya, Kuala Lumpur 50603, Malaysia.

Received: 2 April 2015 Accepted: 22 July 2015

Published online: 14 August 2015

\section{References}

1. N Magdalena, 3GPP A global initiative, 2013. http://www.3gpp.org/LTE

2. $M$ Yang, Y Li, D Jin, L Zeng, X Wu, AV Vasilakos, Software-defined and virtualized future mobile and wireless networks: a survey. J Mobile Netw Appl 20(1), 4-18 (2014). doi:10.1007/s11036-014-0533-8

3. X Zhang, Y Zhang, F Yan, AV Vasilakos, Interference-based topology control algorithm for delay-constrained mobile Ad hoc networks. IEEE Trans Mobile Comput 14(4), 742-753 (2015). doi:10.1109/TMC.2014.2331966

4. B Sahoo, Performance Comparison of packet scheduling algorithms for video traffic in LTE cellular network. Int J Mobile Netw Commun Telematics 3(3), 9-18 (2013)

5. P P Demestichas, VG Stavroulaki, LMI Papadopoulou, AV Vasilakos, ME Theologou, Service configuration and traffic distribution in composite radio environments. IEEE Transactions on System Management Cybernetics. Part C: Applications and Reviews, 34(1), 69-81 (2004). doi:10.1109/TSMCC.2003.818500

6. YS Yen, HC Chao, RS Chang, AV Vasilakos, Flooding-limited and multiconstrained QoS multicast routing based on the genetic algorithm for MANETs. Math Comput Model 53(11-12), 2238-2250 (2011). doi:10.1016/j.mcm.2010.10.008

7. AV Vasilakos, C Ricudis, K Anagnostakis, W Pedryca, A Pitsillides, Evolutionaryfuzzy prediction for strategic QoS routing in broadband networks, in Proceedings of IEEE International Conference Fuzzy System IEEE World Congress Computational Intelligence, vol. 2, 1998, pp. 1488-1493. doi:10.1109/FUZZY.1998.686339

8. AA Helen Tang, AV Vasilakos, FR Yu, VCM Leung, A survey of security challenges in cognitive radio networks: solutions and future research directions. Proc IEEE 100(12), 1558-2256 (2012). doi:10.1109/JPROC.2012.2219194

9. D Lopez-Perez, X Chu, AV Vasilakos, H Claussen, Power Minimization based resource allocation for interference mitigation in OFDMA femtocell networks. IEEE J Sel Areas Commun 32(2), 333-344 (2014). doi:10.1109/JSAC.2014.141213 
10. F Capozzi, G Piro, LA Grieco, G Boggia, P Camarda, Downlink Packet scheduling in LTE cellular networks: Key design issues and a survey. IEEE Trans Veh Technol 15(2), 678-700 (2012). doi:10.1109/SURV.2012.060912.00100

11. G Piro, LA Grieco, G Boggia, F Capozzi, P Camarda, Simulating LTE Cellular systems: an open source framework. IEEE Trans Veh Technol 60(2), 498-513 (2011). doi:10.1109/TVT.2010.2091660

12. E Dahlman, S Parkvall, J Sköld, 4G: LTE/LTE-Advanced for Mobile Broadband (Elsevier Ltd., UK, 2014), p. 321-346.

13. PBF Duarte, ZM Fadlullah, AV Vasilakos, N Kato, On the partially overlapped channel assignment on wireless mesh network backbone: a game theoretic approach. IEEE J Sel Areas Commun 30(1), 119-127 (2012). doi:10.1109/JSAC.2012.120111

14. P Li, S Guo, S Yu, AV Vasilakos, CodePipe: an opportunistic feeding and routing protocol for reliable multicast with pipelined network coding, in Proceedings of IEEE INFOCOM, 2012, pp. 100-108. doi:10.1109/INFCOM.2012.6195456

15. IE Kassotakis, ME Markaki, AV Vasilakos, A hybrid genetic approach for channel reuse in multiple access telecommunication networks. IEEE J Sel Areas Commun 18(2), 234-243 (2000). doi:10.1109/49.824804

16. J Max Magalhães Hofmann, P Roberto Guardieiro, A Downlink Scheduling based on Earliest Deadline First Discipline for Real-Time Traffic in LTE Networks. Int Work Telecommun. 1-7 (2013). http://www.inatel.br/iwt2013/ index.php?option=com_content $\&$ view $=$ article\&id $=84$

17. CK Chakravarthy, PVGD Prasad Reddy, Modified Queue-based exponential rule scheduler for improved QoS in OFDMA. Int J UbiComp 1(2), 34-43 (2010)

18. D Lopez-Perez, X Chu, AV Vasilakos, H Claussen, On Distributed and coordinated resource allocation for interference mitigation in self-organizing LTE networks. IEEE/ACM Trans Netw 21(4), 1145-1158 (2013). doi:10.1109/TNET.2012.2218124

19. T Jiang, H Wang, AV Vasilakos, QoE-driven channel allocation schemes for multimedia transmission of priority-based secondary users over cognitive radio networks. IEEE J Sel Areas Commun 30(7), 1215-1224 (2012). doi:10.1109/JSAC.2012.120807

20. EM Ang, KK Wee, YH Pang, SH Lau, Two-level scheduling framework with frame level scheduling and exponential rule in wireless network, in Proceedings of International Conference on Information Science and Applications (ICISA 2014), 2014, pp. 47-50. doi:10.1109/ICISA.2014.6847476

21. A Mae, WK Kwee, PY Han, OS Yin, A Performance analysis on enhanced Two-level scheduling framework for real-time traffic in LTE. Acad J Sci 3(3), 213-222 (2014)

22. A Alfayly, I-H Mkwawa, L Sun, E Ifeachor, QoE-based performance evaluation of scheduling algorithms over LTE, in 2012 IEEE Globecom Work, 2012, pp. 1362-1366. doi:10.1109/GLOCOMW.2012.6477781

23. J-G Choi, S Bahk, Cell-throughput analysis of the proportional fair scheduler in the single-cell environment. IEEE Trans Veh Technol 56(2), 766-778 (2007). doi:10.1109/TVT.2006.889570

24. G Piro, N Baldo, M Miozzo, An LTE module for the ns-3 network simulator, in Proceedings of the 4th International ICST Conference on Simulation Tools and Techniques, 2011, pp. 415-422. doi:10.4108/icst.simutools.2011.245571

25. G Piro, LA Grieco, G Boggia, P Camarda, A Two-level scheduling algorithm for QoS support in the downlink of LTE cellular networks, in Proceedings of European Wireless Conference (EW), 2010, pp. 246-253. doi:10.1109/EW.2010.5483423

26. D Singh, P Singh, Radio Resource scheduling in 3GPP LTE : a review. Int J Eng Trends Technol (IJETT) 4(6), 2405-2411 (2013)

27. LIU Gan, Z Xin, ZHU Guang-xi, A scheduling algorithm for maximum throughput based on the link condition in heterogeneous network. J Commun Comput 4(3), 33-37 (2007)

28. H Ekstrom, Ericsson, QoS Control in the 3GPP evolved packet system. IEEE Commun Mag 47(2), 76-83 (2009). doi:10.1109/MCOM.2009.4785383

29. N Saed, K Wee, TH Liew, SY Ooi, Video and voice transmission over LTE networks, in Proceedings of IEEE International Conference on Control System, Computing and Engineering, 2013, pp. 259-263. doi:10.1109/ICCSCE.2013.6719970

30. G Aiyetoro, F Takawira, A Cross-layer based packet scheduling scheme for multimedia traffic in satellite LTE networks, in Proceedings of 6th International Conference on New Technologies, Mobility and Security (NTMS), 2014, pp. 1-6. doi:10.1109/NTMS.2014.6813994

31. R Basukala, HAM Ramli, K Sandrasegaran, Performance analysis of EXP/PF and M-LWDF in downlink 3GPP LTE system, in Proceedings of First Asian
Himalayas International Conference on Internet (AH-ICl 2009), 2009, pp. 1-5. doi:10.1109/AHICI.2009.5340336

32. A Biernacki, K Tutschku, Comparative Performance study of LTE downlink schedulers. 12th IEEE Int Symp Netw Comput Appl 74(2), 585-599 (2013). doi:10.1007/s11277-013-1308-4

33. B Sadiq, SJ Baek, G De Veciana, Delay-optimal opportunistic scheduling and approximations: the log rule. IEEE/ACM Trans Netw 19(2), 405-418 (2011). doi:10.1109/TNET.2010.2068308

34. N Marcus, SOS Mathematics. (MathMedics, LLC), http://www.sosmath.com/ algebra/logs/log4/log4.html

35. G Piro, LTE Simulator. (The LTE Simulator, 2007), http://telematics.poliba.it/ index.php/en/lte-sim.

36. B Sadiq, SJ Baek, G de Veciana, Delay-optimal opportunistic scheduling and approximations: the Log rule. IEEE/ACM Trans Netw 19(2), 405-418 (2010). doi:10.1109/TNET.2010.2068308

37. S Ali, M Zeeshan, A Naveed, A capacity and minimum guarantee-based service class-oriented scheduler for LTE networks. EURASIP J Wirel Commun Netw 67(1), 1-15 (2013)

38. S Ali, M Zeeshan, A Utility based resource allocation scheme with delay scheduler for LTE service-class support, in Proceedings of Wireless Communications and Networking Conference (WCNC), 2012, pp. 1450-1455. doi:10.1109/NCNC.2012.6214009

39. M Iturralde, A Wei, T Ali-Yahiya, A-L Beylot, Resource Allocation for real time services in LTE networks: resource allocation using cooperative game theory and virtual token mechanism. Wirel Pers Commun 72(2), 1415-1435 (2013). doi:10.1007/s11277-013-1086-z

40. MA Khan, H Tembine, AV Vasilakos, Game dynamics and cost of learning in heterogeneous 4G networks. IEEE J Sel Areas Commun 30(1), 198-213 (2012). doi:10.1109/JSAC.2012.120118

\section{Submit your manuscript to a SpringerOpen ${ }^{\odot}$ journal and benefit from:}

- Convenient online submission

- Rigorous peer review

- Immediate publication on acceptance

- Open access: articles freely available online

- High visibility within the field

- Retaining the copyright to your article

Submit your next manuscript at springeropen.com 